\title{
Los docentes en la sociedad actual: sus creencias y cogniciones pedagógicas respecto al proceso didáctico*
}

\author{
Claudio Díaz L.** \\ Patricia Martínez I. \\ Iris Roa G. \\ María Gabriela Sanhueza J.
}

\begin{abstract}
Resumen: Este artículo examina las diferentes conceptualizaciones del sistema cognitivo de los docentes, desde la perspectiva socioconstructivista. Aborda la cognición como una base conceptual, personal, subjetiva y dinámica que tiene un impacto significativo en la actuación docente y en el proceso de cambio en educación. El proceso de exploración de la cognición docente puede ayudar a enriquecer la conceptualización sobre el proceso didáctico, y, además, puede servir como base para la autoevaluación docente, componente importante del desarrollo profesional en la Educación Superior.
\end{abstract}

Palabras clave: cognición, práctica pedagógica, docente, creencias

\section{Teachers' in the current society: their pedagogical beliefs and cognition about the teaching-learning process}

\begin{abstract}
This paper examines the different conceptualizations of teachers' cognition from a socio-constructivist perspective. It approaches cognition as a conceptual, personal, subjective and dynamic base that has a significant impact on both teachers' performance and behaviour in the classroom, and on the process of change and innovation in Education. Exploring teachers' cognition can certainly enrich the teaching and learning process and can also serve as a basis for professional development in Higher Education.
\end{abstract}

Key words: cognition, pedagogical practices, teacher, beliefs

\footnotetext{
* Este artículo se inserta en el contexto del proyecto FONIDE 91, año 2008, del Fondo de Investigación en Educación del MINEDUC titulado "Los procesos de cognición y actuación docente como ejes articuladores del saber pedagógico y su impacto en el diseño de un modelo de práctica pedagógica reflexiva para la formación inicial docente: Un estudio en algunos establecimientos municipalizados de la octava región”.

** Universidad Católica de la Santísima Concepción. Concepción. Chile. cdiaz@ucsc.cl / pmartine@ucsc.cl / iroa@ucsc.cl / gabrielasanhueza@ucsc.cl
} 


\section{Os docentes na sociedade actual: suas crenças e cogniciones pedagógicas com respeito ao processo didáctico}

Resumo: Este artigo examina as diferentes conceptualizaciones do sistema cognitivo dos docentes, desde a perspectiva socioconstructivista. Aborda a cognição como uma base conceptual, pessoal, subjetiva e dinâmica que tem um impacto significativo na actuação docente e no processo de mudança em educação. O processo de exploração da cognição docente pode ajudar a enriquecer a conceptualização sobre o processo didáctico, e, ademais, pode servir como base para a autoevaluação docente, componente importante do desenvolvimento profissional na Educação

Palavras-chave: cognição, prática pedagógica, docente, crenças

Recibido: 09.04.2009

Aprobado: 26.10.2009

$* * *$

En cada aula ocurren eventos que permiten desarrollar una comprensión más profunda del proceso de enseñanza-aprendizaje. Cada aula es un escenario en el que interactúa una serie de variables didácticas que hacen de la enseñanza y el aprendizaje un acto complejo. Los docentes se relacionan con los estudiantes, los objetivos, los métodos, las actividades, los materiales, la evaluación y el contexto. Sin embargo, un acercamiento a la comprensión de dicha complejidad se puede lograr al abordar las creencias y las actuaciones docentes para interpretar la multitud de variables que ocurren en el fenómeno de la transposición didáctica.

La idea de explorar el fenómeno didáctico desde las creencias y actuaciones de los docentes, se relaciona con la alta cuota de subjetividad y complejidad que dicho fenómeno reviste. La actuación del docente y su concepción del proceso de enseñanza y aprendizaje, se comprende al entrar al entramado de creencias que sostiene su praxis pedagógica.

Los estudios acerca de las creencias de los docentes y sus actuaciones de aula han aumentado considerablemente durante este último tiempo. Esta información, sin duda, resulta relevante si se pretende dar respuesta a los nuevos paradigmas educativos que han impactado la praxis pedagógica en muchas aulas. Un proceso de reforma educacional implica mucho más que un cambio de prácticas pedagógicas. "Una reforma educacional exitosa implica comprender y considerar el sistema de creencias y actuaciones del docente. El docente debería ser considerado como una persona que está aprendiendo activamente y que construye sus propias interpretaciones" (Bucci, 2002 y Mae, 2004). Al comprender la relevancia del docente en el proceso de enseñanza-aprendizaje, se entiende la necesidad de que los profesores hagan cambios fundamentales sobre cómo enseñan y cómo conciben la enseñanza. El docente es una persona que experimenta situaciones de enseñanza-aprendizaje y les otorga significado personal a través de la reflexión. 
Para comprender mejor el proceso de enseñanza-aprendizaje, es preciso indagar tanto en lo que sucede en las clases como en las fuerzas que operan en profundidad bajo las actuaciones visibles de los docentes. Resulta importante preguntarse ¿Qué piensan los docentes acerca de los contenidos que enseñan? ¿Cómo creen que se deben enseñar y cómo los enseñan? ¿Cómo creen que aprenden sus estudiantes?

El énfasis en el docente se debe a que las decisiones que él o ella toma, están relacionadas con un sistema propio de creencias que le permite afrontar la complejidad de su trabajo. Hasta cierto punto, el cambio o la innovación educativa significan necesariamente, un cambio en las creencias de los docentes. Las contribuciones más significativas en el campo de la investigación educativa están relacionadas con las actuaciones de aula. Sin duda, una mayor comprensión del sistema de creencias del docente contribuye significativamente a mejorar la efectividad educativa. "Los sistemas de creencias son dinámicos en su naturaleza, sufren cambios y reestructuraciones, a medida que los individuos evalúan sus creencias con sus experiencias” (Camps, 2002: 197, Crux, 1989: 39 y Fazio: 2005:64).

Existe la idea generalizada de que las creencias de los docentes son los mejores indicadores de las decisiones que ellos toman durante el transcurso de su vida pedagógica cotidiana. Este énfasis en los sistemas de creencias ha sido explotado por los investigadores en educación, al momento que intentan comprender la naturaleza del proceso de enseñanza-aprendizaje en el aula. Existe gran cantidad de literatura que sugiere que las creencias que sostienen los docentes tienen un impacto considerable tanto en sus percepciones como en sus juicios, los que, a su vez, afectan su actuación en el aula (Biddle, et al., 2000). Además, estos sistemas de creencias son una parte esencial para mejorar la formación profesional y, por ende, la efectividad docente. Dichas creencias pueden ser influenciadas tanto por su formación profesional como también por otros factores tales como su cultura y sus teorías personales sobre la enseñanza-aprendizaje del inglés.

Los docentes que están mejor informados acerca de la naturaleza de la enseñanza-aprendizaje son capaces de evaluar su crecimiento profesional y los aspectos de sus actuaciones pedagógicas que necesitan cambiar. Además, cuando la reflexión crítica se visualiza como un proceso sistemático que forma parte de la enseñanza y el aprendizaje, ella permite que los docentes se sientan más seguros cuando se adscriben a diferentes opciones pedagógicas y evalúan sus efectos. El éxito o fracaso de las innovaciones educativas depende, en gran parte, de la forma en que los diferentes actores educativos interpretan, redefinen, filtran y dan forma a los cambios propuestos. La manera en que estos procesos mediadores operan, depende de lo que los docentes piensan, sienten y hacen, y de sus concepciones acerca de las diferentes dimensiones de lo educativo.

Por ello, las acciones dirigidas al mejoramiento de los procesos de enseñanza- aprendizaje en la educación superior no pueden desconocer las mediaciones cognitivas, sociales y culturales que vehiculizan y posibilitan 
los cambios educativos. Los procesos deliberados o intencionales de innovación suponen prever estrategias que articulen cambios en los ámbitos subjetivos y objetivos. El ámbito subjetivo supone el cambio de las creencias de los actores, desde las cuales interpretan y adaptan las innovaciones. El ámbito objetivo refiere a las prácticas que son objeto de transformación: objetivos, contenidos de enseñanza, estrategias metodológicas, materiales curriculares, enfoques y prácticas de evaluación.

En este sentido, cuando el docente reflexiona acerca de su rol en el proceso de enseñanza-aprendizaje toma conciencia de sus creencias y actuaciones. Esta acción implica que asume su responsabilidad en dicho proceso. A su vez, el docente tiene la necesidad de sus pares, a fin de comprender sus propias experiencias y enriquecerse del otro. La literatura relacionada con este tema señala que no existe una única forma de enseñar. No se ha encontrado la metodología correcta que se debe seguir para ser un docente eficaz (Chevallard, 1991; Monereo, et al., 2004; Sanjurjo, 2002).

\section{Los procesos cognitivos de los docentes}

El estudio de la vida de los docentes puede entregar un abanico de perspectivas acerca de los procesos de reforma, restructuración y reconceptualización pedagógica (Freeman, 2002, Goodson \& Numan, 2002 y Maclellan \& Soden, 2003). Si las creencias de los docentes no se toman en cuenta, lo más probable es que se obstruyan los nuevos procesos de cambio o reformas. Si los docentes no son considerados en las nuevas iniciativas, su centralidad en el proceso de enseñanza-aprendizaje puede constituir un obstáculo para la esencia de las reformas educativas.

\section{La cognición y el contexto}

Las prácticas pedagógicas de los docentes están también determinadas por las realidades sociales, psicológicas y contextuales de la institución y del aula (Andrews, 2003, Bailey, et al., 2001, Johnstone, 1999, Maclellan \& Soden, 2003 y Richards, 2001). Estos factores involucran a los apoderados, los requerimientos de las autoridades, la institución, la sociedad, los principios curriculares, la distribución de las salas y de la institución, las políticas institucionales, los colegas, el uso de exámenes estandarizados, estudiantes no motivados, un currículo rígido, los docentes más antiguos y la disponibilidad de recursos didácticos. Estos factores pueden influir en la habilidad del docente para adoptar actuaciones pedagógicas que reflejen sus creencias. En esta línea, las condiciones laborales difíciles también pueden influir en lo que hace el docente y entrar en conflicto con su cognición. Por ejemplo, existe evidencia que muestra cómo el entusiasmo inicial de un docente puede verse afectado por las exigencias organizacionales que escapan a su control. Estos factores pueden desincentivar la experimentación y la innovación pedagógica, y promover la estrategia segura y 
confiable de apegarse a los enfoques y materiales prescritos. Con esto, los docentes se alinean con las características del contexto de enseñanza.

\section{La cognición y la experiencia docente}

La cognición no sólo estructura lo que los docentes hacen, sino que ésta también se estructura por las experiencias que los docentes acumulan. Muchos docentes se refieren a su experiencia como una entidad única, personal y propia. Es una historia personal de conocimiento e información que se obtiene a través del ensayo y el error y se relaciona con las ideas pedagógicas que son efectivas bajo determinadas circunstancias (Menin, 2001; Pintor \& Vizcarro, 2005).

Se ha observado, además, que los docentes con mayor experiencia muestran una mayor preocupación en relación con los contenidos didácticos, mientras que los docentes con menor experiencia se focalizan mayoritariamente en la gestión de aula (Frabboni, 2001). Esto sugiere que con la experiencia, los docentes aprenden a automatizar las rutinas relacionadas con la gestión de aula; por lo tanto, pueden focalizarse en el contenido que deben enseñar. Los docentes con mayor experiencia, por otra parte, hacen un mayor uso de la improvisación. Esto significa que a medida que el docente adquiere mayor experiencia, se apega en menor grado a las programaciones didácticas realizadas antes de una clase (Andrés \& Echeverri, 2001, Maclellan \& Soden, 2003 y Vásquez \& Timmerman, 2000). Es interesante constatar que los docentes noveles muestran menos habilidad en las siguientes áreas:

- el conocimiento del contenido desde la perspectiva del estudiante.

- el conocimiento profundo del contenido.

- el conocimiento de cómo presentar el contenido de manera apropiada.

- el conocimiento de cómo integrar el aprendizaje con objetivos curriculares más amplios.

\section{Características de las creencias de los docentes}

Barry \& Ammon (1996), Goodson \& Numan, (2002), Kennedy (2002), Levin (2001), Muchmore (2004) Pajares (1992), Richards \& Lockhart (1998) y Tillema (1998) identifican, aunque no compartidas por todos los autores, las siguientes características de las creencias:

- se forman tempranamente y tienden a autoperpetuarse, y persistir frente a la contradicción causada por el tiempo, la razón, la escolaridad o la 
experiencia.

- las personas desarrollan un sistema de creencias que alberga a todas aquellas adquiridas mediante el proceso de transmisión cultural.

- el sistema de creencias tiene una función adaptativa que ayuda a las personas a definir y comprender el mundo y a ello/as mismo/as.

- el conocimiento y las creencias están intrínsicamente relacionados; sin embargo, la naturaleza afectiva, evaluativa y episódica de las creencias hace que ellas se conviertan en un filtro mediante el cual se interpretan nuevos fenómenos.

- los procesos de pensamiento pueden muy bien ser los precursores y creadores de las creencias; no obstante, el efecto filtro de las creencias redefine, distorsiona y reestructura el procesamiento posterior de la información.

- las creencias epistemológicas tienen un rol fundamental en la interpretación del conocimiento y monitoreo cognitivo.

- las creencias se priorizan, según las conexiones o relaciones, con otras creencias u otras estructuras cognitivas y afectivas.

- entre más temprano se incorpora una creencia en la estructura de creencias, su modificación resulta más difícil.

- el cambio de las creencias durante la adultez es un fenómeno relativamente raro.

- las creencias son instrumentales en la definición de tareas y la selección de herramientas cognitivas con las cuales interpretar, planificar y tomar decisiones con respecto a las tareas; por lo tanto, las creencias tienen un rol fundamental en la definición de un comportamiento y la organización del conocimiento y la información.

- las creencias influyen en la percepción, pero no son un camino confiable para acceder a la naturaleza de la realidad.

En consecuencia, las creencias se definen como un sistema en el cual subyacen constructos que el docente usa cuando piensa, evalúa, clasifica y guía su actuación pedagógica. Las creencias de los docentes responden casi siempre al sentido común y son de naturaleza tácita. No son necesariamente coherentes; sino que más bien se consideran como dinámicas y sujetas al cambio y a la reformulación gradual, a pesar de que otros autores (Kember, 2001 y Lonning \& Sovik, 1987) consideren que el cambio de una creencia es relativamente raro. El proceso de reformulación podría ocurrir cuando las creencias se explicitan y constituyen un desafío directo para el docente o cuando ellas son invalidadas por experiencias pasadas. 
El entramado intelectual del profesor se denomina teorías, concepciones, pensamientos, creencias, representaciones, conocimientos o saberes. Estos sistemas conceptuales se originan y se desarrollan en la experiencia y se abstraen de un conjunto de experiencias almacenadas en la memoria; están determinadas por los contextos educativos; son eclécticos, desorganizados, parciales y sin cohesión; no tienen por qué reproducir significados estandarizados o comúnmente aceptados y, a diferencia del conocimiento científico, tienen un carácter subjetivo, popular y temporal; son personales, particulares y específicos; se mantienen normalmente implícitos; a pesar de su resistencia al cambio, pueden evolucionar por influencia de nuevas experiencias y procesos de debate (Camps, 2001: 210).

Para Crookes (2003), Hamel (2003), Harmer (1998) Moll (1993), Tillema, (1998) y Williams \& Burden (1999) las creencias de los docentes influyen en su actuación más que los conocimientos disciplinarios que ellos poseen. Las creencias tienden a estar limitadas culturalmente, a formarse en una época temprana de nuestra vida y a ser resistentes al cambio. Las creencias acerca de la enseñanza parecen estar bien asentadas cuando un estudiante llega a la universidad. Ellas están íntimamente relacionadas con lo que se cree que se sabe, pero ofrecen un filtro eficaz que discrimina, redefine, distorsiona o modifica el pensamiento y el procesamiento de la información posteriores.

Las creencias de los docentes respecto a lo que es la enseñanza afecta a toda su actuación dentro del aula, tanto si estas creencias son implícitas como explícitas (González, et al., 2001 y Scovel, 2001). Aunque un docente actúe de forma espontánea o por costumbre, sin pensar en la acción, dichas actuaciones surgen de una creencia profundamente enraizada que puede que nunca se haya explicitado. En este sentido, las creencias profundamente enraizadas que tienen los docentes sobre la forma en que se aprende un contenido impregnarán sus actuaciones en el aula más que el método concreto que estén obligados a adoptar o el texto que utilizan.

Richards (1999), Roberts (2002) y Tsui, (2003) señalan que estudiar las creencias de los docentes implica explorar el lado oculto de la enseñanza. Esto se sustenta en dos procesos socio-cognitivos. Uno de ellos está relacionado con cómo los docentes aprenden a enseñar. El otro proceso implica el tema epistemológico relacionado con cómo los docentes saben lo que deben para hacer lo que hacen. El primer punto examina lo que se conoce como el aprendizaje del docente, mientras que el segundo se relaciona con el conocimiento docente. Estos dos procesos están interrelacionados y se comunican entre sí. De hecho se podría discutir que es muy difícil conceptualizar cómo aprenden los docentes sin alguna noción de lo que están aprendiendo.

Camps (2001), Graves (2000) y Woods (1996) proponen el concep- 
to de sistema denominado BAK, formado por creencias (beliefs), presuposiciones (assumptions) y conocimientos (knowledge). Se genera así un sistema de tres elementos que Camps (2001) llama creencias, representaciones y saberes (CRS). Se entiende como creencias los aspectos cognitivos no necesariamente estructurados y con fuerte dimensión personal; las representanciones serían aspectos cognitivos tomados de una dimensión social, es decir, compartidos por el grupo de profesores; los saberes tendrían un componente de conocimiento establecido de forma convencional y relativo a cuestiones de enseñanza-aprendizaje. A pesar de estas distinciones estos conceptos están articulados entre sí, no se excluyen, forman un continuum y se traslapan fácilmente.

La comprensión de la estructura de las creencias de los docentes es esencial para mejorar su formación profesional y prácticas pedagógicas. El estudio de las creencias es uno de los constructos psicológicos más importantes para la formación de docentes (Freeman, 2002, Levy \& Ammon, 1996, Pajares, 1992 y Tillema, 1998). Como un constructo global, las creencias no se prestan fácilmente para la investigación empírica. Muchos investigadores consideran las creencias como un misterio que nunca podrá ser claramente definido ni tampoco de utilidad para la investigación. Sin embargo, cuando las creencias se operacionalizan específicamente y se utiliza un diseño de investigación apropiado, los estudios de las creencias pueden ser muy relevantes.

\section{Las creencias y el conocimiento}

Tratar de distinguir entre conocimiento y creencias es una tarea gigantesca. Al tratar de diferenciar entre conocimiento y creencias se están utilizando dos términos diferentes para referirse al mismo fenómeno. Las creencias son conocimiento anclado; ellas muestran el conocimiento que es más valioso para el docente y que ha funcionado en la actuación pedagógica. Carusetta \& Cranton (2005) y Day (1993) identifican cuatro características de las creencias: (1) la presunción existencial, (2) la alternancia, (3) la carga afectiva y evaluativa y (4) la estructura de la memoria episódica.

Las presunciones existenciales se refieren a aquellas verdades personales que cada persona tiene; son aquellas creencias respecto a la realidad social y física. Cuestionar estas creencias es cuestionarse uno mismo, la propia sanidad mental (Chacón, 2008; Libedinsky, 2001; Prieto, 2008). Como tal, estas creencias son profundamente personales y no se ven afectadas por la persuasión. Estas creencias se forman por accidente, por experiencias intensas o por una secuencia de eventos. Por ejemplo, un docente puede creer que los estudiantes que reprueban una asignatura son flojos. Las presunciones existenciales son entidades inmutables y van más allá del control individual o el conocimiento.

En algunas circunstancias, las personas, por diferentes razones, intentan crear una situación ideal, alternativa que difiere de la realidad. Por 
ejemplo, se puede mencionar el caso de una docente que, debido a su experiencia traumática como estudiante, intenta crear un ambiente ideal en el aula, con el cual ella había soñado cuando era estudiante. Como existía una inconsistencia entre su actuación pedagógica y los procedimientos efectivos para el aula, sus clases presentaban constantes quiebres y frecuentemente no tenían un cierre.

Golombek (1998) y Sanjurjo (2002) señalan que las creencias tienen un componente afectivo y evaluativo más fuerte que el conocimiento. El afecto opera independiente de la cognición, la cual está asociada al conocimiento. El conocimiento de un área se diferencia de los sentimientos respecto a otra área. Los docentes enseñan el contenido de un curso según los juicios que tengan respecto al contenido en sí. La combinación entre afecto y evaluación puede determinar la energía que los docentes le ponen al desarrollo de una actividad.

Ben-Peretz (2002) y Milicia, et al. (2004), además, señalan que el conocimiento se almacena semánticamente, mientras que las creencias residen en la memoria episódica, producto de las experiencias o fuentes culturales de la transmisión de conocimiento. Las creencias recogen su fuerza de los episodios o eventos previos que influencian la compresión de eventos posteriores. Por ejemplo, los esfuerzos de la docente por crear un ambiente agradable en su aula tenían su origen en sus recuerdos como estudiante. Otros investigadores han descubierto que los docentes son influenciados por imágenes creadas por eventos pasados. Estas imágenes funcionan como pantallas intuitivas que filtran la nueva información.

Una experiencia crucial en la vida de un docente o algún profesor importante en su formación profesional genera una memoria episódica detalladamente rica que sirve posteriormente como inspiración o marco de referencia para sus futuras actuaciones pedagógicas. La memoria episódica se forma producto de las experiencias vividas con antiguos docentes, la literatura revisada e incluso la influencia de los medios de comunicación (Cárdenas, et al., 2000, Rodríguez, et al., 2004, Scovel, 2001 y Sendan \& Roberts, 1998).

Los sistemas de creencias, a diferencia de los sistemas de conocimiento, no requieren de un consenso general o grupal con respecto a la validez y pertinencia de las creencias. La ausencia del consenso general implica que los sistemas de creencias son, por naturaleza, más inflexibles, y menos dinámicos que los sistemas de conocimientos. Las creencias son más influyentes que el conocimiento en la determinación de la forma cómo las personas organicen y definan las tareas y problemas, y son predictores más efectivos respecto al comportamiento de un sujeto.

Ben-Peretz (2002), Tsang (2004) y Zanting, et al. (2003) presentan evidencia que indica que al estudiar las creencias de dos docentes de inglés respecto a la enseñanza de la gramática, por ejemplo, es muy importante la confianza que el docente tenga en sus propias creencias, puesto que los 
docentes que muestran un mayor grado de confianza respecto a este punto están más dispuestos a promover la discusión de los puntos gramaticales y a formular reglas espontáneamente en cualquier momento de la dinámica de la clase. No obstante, el docente que tiene menos confianza en sus creencias entrega respuestas más directas que minimizan o difieren la discusión. El factor confianza parece estar relacionado con el nivel del conocimiento del contenido pedagógico del docente. Quien muestra más confianza posee un repertorio bien desarrollado de estrategias metodológicas para la enseñanza de la gramática, mientras que el docente con un menor grado de confianza posee un repertorio limitado de estrategias que son utilizadas de manera poco efectiva.

\section{Consideraciones finales}

El estudio de las creencias de los docentes permite re-examinar el rol del docente en el aula; no desde la perspectiva de un sujeto que es todo en el proceso de enseñanza y aprendizaje, pero sí desde la perspectiva de un sujeto que está en constante aprendizaje y búsqueda y que necesita adquirir y desarrollar herramientas que lo habiliten para continuar su propio proceso de aprendizaje. El docente es en el sistema didáctico otro estudiante más, tal vez, con conocimientos, destrezas, habilidades y actitudes más avanzadas que los estudiantes que comúnmente se conocen.

Cabe preguntarse entonces, ¿cómo un docente que recibe un perfeccionamiento específico y externo, puede transferir directamente lo aprendido a sus estudiantes y al mejoramiento de los procesos de enseñanzaaprendizaje? Es impensable, no considerar que este docente filtra y reinterpreta lo aprendido mediante su sistema de creencias. Cuando los docentes reciben perfeccionamiento, entonces, ¿no será necesario comenzar con la identificación de su sistema de creencias para después determinar qué perfeccionamiento necesita?

El docente es el actor principal al cual se debe apuntar para lograr cambio e innovación en educación. Entiéndase actor principal, no en el sentido prescriptivo y comúnmente difundido como dueño del conocimiento; al contrario, es un actor y agente de cambio clave para el inicio de la innovación y cambio en educación. Todas las otras variables didácticas, a saber, el estudiante, los contenidos, la metodología, etc. pueden ser intervenidas para optimizar el proceso de enseñanza-aprendizaje; sin embargo, poco se puede lograr si es que no se conoce e interviene la variable principal: el docente. Es él o ella quien vehiculiza el cambio o la innovación en educación.

El docente, conciente o inconcientemente, y el contexto socioeducativo sitúan al docente en una relación de poder, que lo lleva a relacionarse con todas las variables didácticas, desde su sistema de creencias y concepción de mundo. Esta relación de poder será ejercida con un mayor o menor control, dependiendo de cómo el docente conceptualiza su 
rol en el proceso de enseñanza-aprendizaje.

El docente debe, continuamente, aprender y re-aprender a enseñar, puesto que durante su vida profesional se verá enfrentado a diversos contextos socio-educativos que presentarán complejidades distintas. Este aprendizaje no finaliza cuando el estudiante egresa de la Universidad, sino que, por el contrario, el aprendizaje se complementa, profundiza y se articula durante toda su vida académica. Este aprendizaje es extremadamente personal y ayuda a que el docente comprenda su actuación pedagógica. El docente no puede aislarse de su contexto; su contexto lo lleva a actuar y determina su actuación.

Los docentes están conscientes de sus creencias; sin embargo, la complejidad del proceso de enseñanza-aprendizaje, les impide, muchas veces, que ellas se condigan con su actuación en el aula. El docente sostiene una creencia respecto a alguna variable didáctica del proceso de enseñanza y aprendizaje. Esta creencia puede provenir de la teoría; sin embargo, la realidad educativa la confronta y, es aquí, donde el docente atraviesa por una serie de tensiones que, en ocasiones, no sabe cómo resolver. En este sentido, la reflexión sistemática y la discusión constante con los pares y diferentes actores del proceso educativo, deberían constituir dos estrategias importantes de perfeccionamiento docente.

Por otro lado, puesto que las prácticas docentes están social y contextualmente mediadas, los docentes están fuertemente influenciados por sus experiencias previas como estudiantes, su formación profesional, y sus creencias personales sobre la enseñanza efectiva. Más aún, cuando los docentes intentan cambiar su sistema de creencias y practicas puede ocurrir un desequilibrio entre dichas creencias y prácticas. De esta forma, el docente puede experimentar sentimientos de incomodidad y frustración a medida que intenta alinear sus prácticas docentes con un sistema de creencias en expansión o alterado.

El sistema de creencias no cambia fácilmente; sin embargo, existen dos fuerzas significativas que pueden ocasionar cambios en los docentes. Primero, cuando la cultura prevaleciente comienza a cambiar persistentemente sus valores, los docentes, en este punto, comienzan a mover sus pensamientos en una dirección similar. Segundo, los docentes parecen adaptar sus sistemas de creencias para acomodarse a las nuevas realidades.

Investigar los pensamientos y conocimientos de profesores/as significa comprender las concepciones, creencias, dilemas, teorías que gobiernan la práctica profesional e identificar los procesos que constituyen el aprender a enseñar y las categorías conceptuales en las que se articula el conocimiento básico para desarrollar la actividad profesional de la enseñanza.

El aporte más significativo de los estudios respecto de las creencias de los docentes es descubrir que el comportamiento profesional de los pro- 
fesores está guiado por un complejo sistema de conocimientos, creencias, valores, teorías, principios que regulan su actividad. De esta constatación no se deduce que exista una total correspondencia entre el sistema conceptual que poseen los profesores y su actividad en el aula. Más bien se constatan discrepancias entre lo que se dice y lo que se hace, lo que se piensa y de lo que se informa. Ciertamente estas discrepancias pueden servir de estimulo para refinar los conceptos y estrategias de indagación sobre cuestiones tan resistentes.

La imagen que de los profesores emerge del esfuerzo investigador realizado desde el paradigma del pensamiento y conocimiento de los profesores es la del profesor como un profesional reflexivo. Una imagen contrapuesta a la predominante del profesor como un técnico, fundamentalmente alimentada, por los estudios conductistas sobre el comportamiento del docente.

Los estudios sobre el pensamiento de los profesores han ayudado a ampliar y profundizar las ideas sobre las características propias de la actividad profesional del docente gracias a la disponibilidad de un mayor acceso a la interpretación que los propios profesores hacen de ella. A partir de ellos se dispone de un rico bagaje de constructos mediante los cuales se pretende comprender las características propias del conocimiento profesional que se construye en la práctica pedagógica y examinar la coherencia entre el hacer y el decir, profundizando en el currículum oculto de la enseñanza. 


\section{Bibliografía}

Andrés, G. \& Echeverri, P. (2001), Pensamiento docente y práctica pedagógica. Una investigación sobre el pensamiento práctico de los docentes. Magisterio. Bogotá.

Andrews, Stephen (2003), “Just like instant noodles': L2 teachers and their beliefs about grammar pedagogy”. Teachers and Thinking: Theory and Practice, 9, 351-370.

Bailey K.; Curtis, A. \& Nunan, D. (2001), Pursuing professional development. Newbury House. Teacher Development. Boston.

Barry, Ben \& Ammon, Patricia (1996), "A longitudinal study of the development of teachers' pedagogical conceptions: The case of Ron". Teacher Education Quarterly, 5, 5-25.

Ben-Peretz, Michael (2002), "Retired teachers reflect on learning from experience". Teachers and Thinking: Theory and Practice, 8, 312-324.

Biddle, B.; Good, T. \& Goodson, I. (2000), La enseñanza y los profesores I. La profesión de enseñar. Paidós. Buenos Aires.

Bucci, Patricia (2002), Teacher knowledge, beliefs and practices of classroom assessment: From the perspective of five elementary teachers. Tesis doctoral, University of Toronto, Toronto.

Camps, A. (2002), El aula como espacio de investigación y reflexión. Investigaciones en didáctica de la lengua. Graó. Barcelona.

Cárdenas, A.; Rodríguez, A. \& Torres, R. (2000), El maestro protagonista del cambio educativo. Magisterio. Bogotá.

Carusetta, Elena \& Cranton, Patrick (2005), "Nurturing authenticity: a conversation with teachers". Teaching in Higher Education, 10, 285-297.

Chacón, María (2008), "Las estrategias de enseñanza reflexiva en la formación inicial docente”. Educere.12. 41, 277-287.

Chevallard, Y. (1991), La transposición didáctica. Del saber sabio al saber enseñado. Aique. Buenos Aires.

Crookes, G. (2003), A practicum in Tesol. Professional development through teaching practice. Cambridge Language Education. Cambridge.

Crux, S. (1989), The congruence of teacher beliefs and practice. Tesis doctoral, University of Toronto, Toronto.

Day, Richard (1993), "Models and the knowledge base of second language 
teacher education”. University of Hawai'I Working Papers in English as a Second Language, 11, 1-13.

Fazio, E. (2005), Exploring teachers' beliefs and knowledge about scientific inquiry and the nature of science: A collaborative action research. Project. Tesis doctoral, University of Toronto, Toronto.

Frabboni, F. (2001), El libro de la pedagogía y la didáctica I: la educación. Editorial popular. Madrid.

Freeman, Donald (2002), “The hidden side of the work: Teacher knowledge and learning to teach. A perspective from North American educational research on teacher education in English language teaching”. Language Teaching. The International Abstracting Journal for Language Teachers and Applied Linguists, 35, 1-13.

Golombek, Paula (1998), “A study of language teachers’ personal practical knowledge”. Tesol Quarterly, 32, 447-464.

Gonzáles S.; Río, E. \& Rosales, S. (2001), El Currículum oculto en la escuela. Lumen Humanitas. Buenos Aires.

Goodson, Ian. \& Numan, Ulises (2002), “Teacher’s life worlds, agency and policy contexts”. Teachers and Thinking: Theory and Practice, 8, 269-277.

Graves, K. (2000), Designing language courses. Heinle and Heinle. Boston.

Hamel, Frank (2003), “Teacher understanding of student understanding: Revising the gap between teacher conceptions and students' ways with literature”. Research in the Teaching of English. 38, 49-84.

Harmer, J. (1998), How to teach English. Longman. England.

Johnstone, Reynold (1999), "Research on language learning and teaching: 1997-1998”. Language Teaching. The International Abstracting Journal for Language Teachers and Applied Linguists, 32, 137-156.

Kember, Dick (2001), “Beliefs about knowledge and the process of teaching and learning as a factor in adjusting to study in higher education”, Studies in Higher Education, 26, 205-221.

Kennedy, Myriam (2002), “Knowledge and teaching”. Teachers and Thinking: Theory and Practice, 8, 355-370.

Levin, Bart (2001), "Lives of teachers: Update on a longitudinal case study”. Teacher Education Quarterly, 7, 29-47.

Libedinsky, M. (2001), La innovación en la enseñanza. Paidós. Buenos Aires. 
Lonning, A. \& Sovik, N. (1987), Teachers thinking. Perspectives and research. Trondheim: Tapir.

Maclellan, Ellen \& Soden, Rey (2003), “Expertise, expert teaching and experienced teachers' knowledge of learning theory”. Scottish Educational Review, 35, 110-120.

Mae, P. (2004), Exploring the beliefs and attitudes of exemplary technologyusing teachers. Tesis doctoral no publicada, The Pennsylvania State University, Pennsylvania.

Menin, O. (2001), Pedagogía y Universidad. Currículo, didáctica y evaluación. Ediciones Homosapiens. Santa Fe.

Milicia, Berta; Utges, Gael, Salinas, Biell \& San José, Vilma (2004), “Creencias, concepciones y enseñanza en la universidad: Un estudio de caso de desarrollo profesional colaborativo centrado en un profesor de física”. Revista Española de Pedagogía, 229, 377-394.

Moll, L. (1993), Vygotsky y la educacion. Connotaciones y aplicaciones de la psicología sociohistórica en la educación.. Aique. Buenos Aires.

Monereo, C., Castillo, M., Clariana, M., Palma, M. \& Pérez, M. (2004), Estrategias de enseñanza y aprendizaje. Formación del profesorado y aplicación en la escuela. Graó. Barcelona.

Muchmore, J. (2004), A teachers’life. Backalong books. San Francisco.

Pajares, Michael (1992), “Teachers’ beliefs and educational research: cleaning up a messy construct”. Review of Educational Research, 62, 307-332.

Pintor, Manuel \& Vizcarro, Carmen (2005), “¿Cómo aprenden los profesores? Un estudio empírico basado en entrevistas”. Revista Complutense de Educación. Vol. 16 Núm. 2623 - 644.

Prieto, Marcia (2008), “Creencias de los profesores sobre evaluación y efectos incidentales”. Revista de Pedagogía. Vol. 29, № 84. 123-144.

Richards, J. \& Lockhart, C. (1998), Estrategias de reflexión sobre la enseñanza de idiomas. Cambridge University Press. Madrid.

Richards, J. (1999), Language teaching awareness. Cambridge University Press. Cambridge.

Idem (2001), Curriculum development in language teaching. Cambridge University Press. Cambridge.

Roberts, B. (2002), Biographical research. Open University Press. Philadelphia. 
Rodríguez, Constanza; Hernández, Juan, \& Calvo, Patricia (2004), "Pensamiento docente sobre el juego en educación infantil: análisis desde una perspectiva de género”. Revista Española de Pedagogía, 229, 455-466.

Sanjurjo, L. (2002), La formación práctica de los docentes. Reflexión y acción en el aula. Homosapiens. Santa Fe.

Scovel, T. (2001), Learning new languages. A guide to second language acquisition. Heinle and Heinle. Boston.

Sendan, Felicity \& Roberts, John (1998), “Orhan: a case study in the development of a student teacher's personal theories”. Teachers and Thinking: Theory and Practice, 4, 229-244.

Tillema, Hellen (1998), “Stability and change in student teachers' beliefs about teaching”. Teachers and thinking: theory and Practice, 4, 217-228.

Tsang, Walter (2004), “Teachers’ personal practical knowledge and interactive decisions”. Language Teaching Research, 2, 163-198.

Tsui, A. (2003), Understanding expertise in teaching. Cambridge University Press. Cambridge.

Williams, M. \& Burden, R. (1999), Psicología para profesores de idiomas. Cambridge University Press. Madrid.

Woods, D. (1996), Teacher cognition in language teaching: beliefs, decision-making and classroom practice. Cambridge University Press. Cambridge.

Zanting, Albert; Verloop, Noami \& Vermut, John (2003), "How do student teachers elicit their mentor teachers practical Knowledge?”, Teachers and Thinking: Theory and Practice, 9, 197-212. 